\title{
Saxagliptin affects long-bone microarchitecture and decreases the osteogenic potential of bone marrow stromal cells
}

\author{
María Laura Sbaraglini, María Silvina Molinuevo, Claudia Sedlinsky, León Schurman, \\ Antonio Desmond McCarthy*
}

LIOMM, Laboratorio de Osteopatías y Metabolismo Mineral, Facultad de Ciencias Exactas, Universidad Nacional de La Plata,

47 y 115, 1900 La Plata, Argentina

\section{A R T I C L E I N F O}

\section{Article history:}

Received 26 September 2013

Received in revised form

26 December 2013

Accepted 9 January 2014

Available online 28 January 2014

\section{Keywords:}

Bone microarchitecture

Diabetes mellitus

Saxagliptin

Dipeptidyl-peptidase 4

Osteoblasts

Bone marrow stromal cells

\begin{abstract}
A B S T R A C T
Diabetes mellitus is associated with a decrease in bone quality and an increase in fracture incidence. Additionally, treatment with anti-diabetic drugs can either adversely or positively affect bone metabolism. In this study we evaluated: the effect of a 3-week oral treatment with saxagliptin on femoral microarchitecture in young male non-type-2-diabetic Sprague Dawley rats; and the in vitro effect of saxagliptin and/or fetal bovine serum (FBS), insulin or insulin-like growth factor-1 (IGF1), on the proliferation, differentiation (Runx2 and PPAR-gamma expression, type-1 collagen production, osteocalcin expression, mineralization) and extracellular-regulated kinase (ERK) activation, in bone marrow stromal cells (MSC) obtained from control (untreated) rats and in MC3T3E1 osteoblast-like cells. In vivo, oral saxagliptin treatment induced a significant decrease in the femoral osteocytic and osteoblastic density of metaphyseal trabecular bone and in the average height of the proximal cartilage growth plate; and an increase in osteoclastic tartrate-resistant acid phosphatase (TRAP) activity of the primary spongiosa. In vitro, saxagliptin inhibited FBS-, insulin- and IGF1-induced ERK phosphorylation and cell proliferation, in both MSC and MC3T3E1 preosteoblasts. In the absence of growth factors, saxagliptin had no effect on ERK activation or cell proliferation. In both MSC and MC3T3E1 cells, saxagliptin in the presence of FBS inhibited Runx2 and osteocalcin expression, type-1 collagen production and mineralization, while increasing PPAR-gamma expression. In conclusion, orally administered saxagliptin induced alterations in long-bone microarchitecture that could be related to its in vitro downregulation of the ERK signaling pathway for insulin and IGF1 in MSC, thus decreasing the osteogenic potential of these cells.
\end{abstract}

(c) 2014 Elsevier B.V. All rights reserved.

\section{Introduction}

Dipeptidyl-peptidase-4 (DPP4) is a membrane-bound glycoprotein with proteolytic activity that is expressed in different cell types including T-cell lymphocytes, hepatocytes, gastrointestinal epithelial cells, osteoblasts and bone marrow stromal cells (MSC) (Matteucci and Giampietro, 2009; Stanley et al., 2006). In T lymphocytes, DPP4 participates in the immune response, and its inhibition suppresses mitogen-mediated cell proliferation (Kahne et al., 2000; Schon et al., 1985). The specific role of DPP4 in other cell types is less known.

DPP4 is also involved in insulin metabolism, since it shows proteolytic activity against various enteric incretins. Enteroendocrine cells stimulated by different nutrients present in the gut lumen secrete incretins, and these peptide hormones then

\footnotetext{
* Corresponding author. Tel.: + $542214235333 \times 33$; fax: + 542214512426 .

E-mail addresses: mccarthy@biol.unlp.edu.ar, antoniomccarthy@yahoo.com (A.D. McCarthy).
}

stimulate pancreatic insulin production, in a mechanism that could account for $50 \%$ of post-prandial insulin secretion. However, incretins are not only insulinotropic, but have multiple additional effects such as lowering glucagon, increasing satiety and slowing gastric emptying. The plasmatic half-life of incretins is dependent on their proteolysis by extracellular DPP4. Selective DPP4 inhibitors (gliptins) have been developed, and are used as second-line drugs for the postprandial control of glycaemic levels in patients with type 2 Diabetes mellitus (Gagliardino et al., 2008). Saxagliptin is a licensed gliptin that is metabolized by cytochrome P450, and whose metabolite is also an active DPP4 inhibitor (Ali and Fonseca, 2013).

An increasing body of evidence demonstrates the association of type 1 and type 2 Diabetes mellitus with bone abnormalities, including osteopenia, osteoporosis and/or an increased incidence of low-stress fractures, in what has been termed diabetic osteopathy (Janghorbani et al., 2007). These bone alterations are partly induced by a significant decrease in the material properties of diabetic bone tissue (i.e., bone quality) (McCarthy et al., 2013). Additionally, bone quality can secondarily be affected by antidiabetic 
pharmacological treatment (Molinuevo et al., 2010; Rzonca et al., 2004; Sedlinsky et al., 2011). Thus, thiazolidinediones (TZD) promote bone loss and fractures via peroxisome proliferator-activated receptor (PPAR)-gamma dependent mechanisms, whereas the biguanide metformin shows in vitro and in vivo osteogenic effects and can prevent in vivo anti-osteogenic actions of TZD.

The effect of gliptins on bone tissue has not been clearly established to date. In the ovariectomized female rat model of post-menopausal osteoporosis, sitagliptin was found to increase bone mineral density and trabecular volume, although in the same study inactivation of the DPP4 gene had no effect on bone phenotype (Kyle et al., 2011). In a recent meta-analysis, other authors have suggested an overall decrease of fracture risk in diabetic patients treated with DPP4 inhibitors (Monami et al., 2011). However, the specific effect of saxagliptin on bone metabolism has not been published to date, and to our knowledge no randomized controlled trials have been reported to specifically evaluate the effects of saxagliptin treatment on fracture incidence.

In the present study we have evaluated the in vivo effects of oral saxagliptin administration in young male non-type-2-diabetic rats on the microarchitecture of load-bearing bones, the in vitro actions of saxagliptin on MSC isolated from control (untreated) rats, as well as possible mechanisms of action involved.

\section{Materials and methods}

\subsection{Animal treatments}

Three-month-old male Sprague-Dawley rats (190-210 g body weight) were used. All experiments on animals were performed in conformity with the Guide for Care and Use of Laboratory Animals of the National Research Council of the National Academies (Guide, 2011). Approval for animal studies was obtained from the institutional accreditation committee (INIBIOLP's Animal Welfare Assurance No A5647-01). Animals were divided into two groups of 5 animals per group: untreated controls $[C]$ and rats treated for 3 weeks with Saxagliptin (Bristol-Myers Squibb Company, USA) administered in drinking water ( $2 \mathrm{mg} / \mathrm{kg} /$ day) [S] (Fura et al., 2009; Tahara et al., 2009). At the end of all treatments blood samples were obtained, after which the animals were sacrificed by cervical dislocation under anesthesia.

\subsection{Biochemical parameters}

Serum was separated from non-fasting blood samples of all animals. Glucose, triglycerides, cholesterol, transaminases (ALT and AST), alkaline phosphatase (ALP), creatinine and urea were measured in serum samples by commercial kits (Wiener Laboratories, Argentina) with a 2300Plus automatic Chemical Analyzer (Metrolab, Argentina). Serum insulin levels were determined with a rat-specific ELISA kit from ALPCO. No significant differences were observed between groups $C$ and $S$ for all parameters evaluated (data not shown).

\subsection{Histological and histomorphometric evaluation of long bones}

Femoral bones were processed for histological and quantitative histomorphometric analysis (Molinuevo et al., 2010). Briefly, sections were stained either with haematoxylin-eosin (H-E) for routine evaluation; with Alcian Blue $(\mathrm{pH}=3)$ to evaluate the average height of the proximal cartilage growth plate; or with tartrate resistant acid phosphatase (TRAP) histochemistry to specifically identify osteoclasts in the associated primary and secondary spongiosa. Images were analyzed using the Image J program (www.macbiophotonics.ca/imagej) with a Microscope scale plugin. Ten sections were processed for every different stains and for each animal.

In the femoral metaphysis $(2 \mathrm{~mm}$ distal from the proximal growth plate), relative trabecular bone volume was determined as the quotient between the area of trabecular bone and the total area (trabecular bone plus bone marrow cavities). Additionally in the same metaphyseal sections, osteoblastic density was evaluated by counting the number of lining osteoblasts per millimeter of trabecular bone surface ( $\mathrm{H}-\mathrm{E})$; and osteocyte density was assessed by determining the number of osteocytes per square millimeter of trabecular bone $(\mathrm{H}-\mathrm{E})$. In sections corresponding to the primary and secondary spongiosa associated with the proximal growth plate, relative osteoclastic density was calculated as the percentage of positive TRAP area per square millimeter of trabecular bone (Sedlinsky et al., 2011).

\subsection{Osteoblastic cell cultures and MSC isolation}

MSC were obtained from animals of group $C$ as we have described previously (Molinuevo et al., 2010). Briefly, bone marrow cells were collected by flushing femora and tibiae of the animals with Dulbecco's modified essential medium (DMEM)-10\% fetal bovine serum (FBS) (Invitrogen, Buenos Aires, Argentina) under sterile conditions. For comparative purposes, in some experiments the MC3T3E1 mouse calvaria-derived preosteoblastic cell line was used instead of MSC.

In experiments designed to evaluate either the direct effect of saxagliptin on MSC and MC3T3E1 proliferation, or its possible modulation of the action and signaling pathways of growth factors present in FBS, sub-confluent cells were serum-starved overnight and then incubated with 1.5 or $15 \mu \mathrm{M}$ saxagliptin and/or FBS (1\%), insulin $(5 \mathrm{ng} / \mathrm{mL})$ or IGF1 $\left(10^{-8} \mathrm{M}\right)$ for $24 \mathrm{~h}$ (effects on cell proliferation) or $1 \mathrm{~h}$ (effects on signal transduction mechanisms). In other experiments, cells were cultured in an osteogenic medium (DMEM-10\% FBS containing $25 \mu \mathrm{g} / \mathrm{ml}$ ascorbic acid and $5 \mathrm{mM}$ sodium $\beta$-glycerol-phosphate) for a further 15 or 21 days, with or without the different doses of saxagliptin. In vitro doses of saxagliptin ( 1.5 and $15 \mu \mathrm{M}$ ) were chosen in view of the plasmatic concentrations of the drug plus its active metabolite that are attained in the rat after administration of $2.5 \mathrm{mg} / \mathrm{kg}$ saxagliptin (Fura et al., 2009).

\subsection{Evaluation of osteoblastic differentiation}

After submitting MSC or MC3T3E1 cells to 15 days of osteogenic induction, type 1 collagen production was evaluated by Sirius Red staining as previously reported (Molinuevo et al., 2010). Extracellular calcium deposits (mineralization nodules) were measured after 21 days of osteogenic differentiation using Alizarin $\mathrm{S}$ red staining (Molinuevo et al., 2010).

\subsection{Western blot analysis}

Bone cells, with or without a 15-day osteogenic induction and under the experimental conditions indicated above, were subjected to $12 \%$ SDS-PAGE. The following antibodies were used: Runx2 and osteocalcin for evaluation of osteoblastogenesis; PPARgamma to evaluate adipocytic commitment; and total extracellular-regulated kinases (ERK) or phosphorylated-ERK to evaluate insulin and IGF1 signal transduction pathways (Santa Cruz Biotechnology, Santa Cruz, CA, USA). Results were normalized with an anti B-actin antibody (Sigma, St. Louis, MO, USA) (Kanazawa et al., 2008) and quantified using the gel plugin of MBF_Image J (http://www.macbiophotonics.ca). 


\subsection{Statistical analysis}

Results are expressed as the mean \pm S.E.M. Differences between groups were assessed by one-way ANOVA using the Tukey post hoc test. For non-normal distributed data, the non-parametrical KruskalWallis test with Dunn post hoc test was performed, using GraphPad In Stat, version 3.00 (Graph Pad Software, San Diego, CA, USA). $P<0.05$ was considered significant for all statistical analyses.

\section{Results}

\subsection{Effect of saxagliptin treatment on bone microarchitecture and cellularity}

We investigated the effects of a 3-week in vivo oral treatment with saxagliptin (group S) on femoral microarchitecture and cellularity, including its possible action on TRAP activity in the primary and secondary spongiosa as well as on the height of the proximal cartilage growth plate.

Compared to control rats, saxagliptin treatment significantly decreased metaphyseal trabecular bone osteocytic density and osteoblastic number, without affecting relative trabecular area (Fig. 1 and Table 1). We also found that, compared to control rats, saxagliptin treatment significantly increased TRAP activity in the primary spongiosa while decreasing the height of the associated growth plate (Fig. 1 and Table 1), but did not affect TRAP activity in the secondary spongiosa (data not shown).

\subsection{Saxagliptin inhibits cell proliferation induced by mitogens}

The alterations induced in vivo by saxagliptin on bone tissue could be due to direct or indirect actions of this protease inhibitor on bone cells. To address this issue, we performed in vitro experiments to evaluate whether saxagliptin could affect the proliferation of MSC obtained from untreated rats (group C), or of MC3T3E1 cells. We found that the addition of 1.5 or $15 \mu \mathrm{M}$ of saxagliptin to serum-starved cells for $24 \mathrm{~h}$ had no effect on the proliferation of both cell types (Fig. 2A and C, white bar). However, $15 \mu \mathrm{M}$ saxagliptin completely blocked the mitogenic actions of FBS ( $1 \%$ ), insulin ( $5 \mathrm{ng} / \mathrm{mL}$ ) or IGF1 $\left(10^{-8} \mathrm{M}\right)$ after $24 \mathrm{~h}$ (Fig. $2 \mathrm{~A}$ and $\mathrm{C}$ ). We also investigated whether saxagliptin could modulate the phophorylation of ERK, a member of the mitogen-activated protein-kinase family that is a well-known downstream mediator of mitogenic signaling pathways. We found that in serum-starved cells saxagliptin did not affect the phosphorylation of ERK (Fig. 2B and $\mathrm{D}$ ). However, $15 \mu \mathrm{M}$ of saxagliptin completely abolished the phosphorylation of ERK induced by FBS, insulin and IGF1 (Fig. 2B and D).

\subsection{Osteogenic cell differentiation is inhibited by saxagliptin treatment}

In further in vitro experiments, MC3T3E1 pre-osteoblasts and MSC were cultured for 14 or 21 days in osteogenic media (including $10 \% \mathrm{FBS}$ ), in the presence or absence of 1.5 or $15 \mu \mathrm{M}$ saxagliptin. In these culture conditions and in both cell types, saxagliptin inhibited type 1 collagen production after 14 days (Fig. 3A), and decreased the formation of nodules of mineralization after 21 days (Fig. 3B). We also evaluated by Western blot the expression of the early osteogenic transcription factor Runx2, the osteoblast-specific marker osteocalcin and the adipogenic inducer PPAR-gamma, and found that saxagliptin modulated these proteins differentially. Thus, saxagliptin dose-dependently downregulated the expression of Runx2 and osteocalcin (Fig. 3C and D) while increasing the expression of PPAR-gamma (Fig. 3E).

\section{Discussion}

Our present in vivo and in vitro studies provide evidence for the first time of a deleterious action of saxagliptin on bone tissue, and point to an indirect mechanism for this effect.

In young healthy male rats that are no longer in their rapid growth phase, in vivo oral administration of saxagliptin diminished the osteocytic and osteoblastic density of femoral metaphyseal trabecular bone. These changes in bone cellularity, which could be caused by a reduced osteogenic commitment of MSC, might be indicating a lower capacity for bone maintenance at a nano- and micrometric scale. In vivo administration of saxagliptin also decreased the average height of the proximal cartilage growth plate and increased osteoclastic TRAP activity in its associated primary spongiosa (without changes in the secondary spongiosa). However, the possible effect of saxagliptin on growth plates should not be an issue in clinical practice in view of the age of patients for whom this DPP4 inhibitor is usually prescribed.

Patients with long-standing Diabetes mellitus frequently show a progressive decrease in the quality of their bone tissue, which is partly due to decreased bone remodeling and diminished osteoblastic bone-forming capacity associated with this disease. This is particularly relevant for weight-bearing bones, since it results in weakened mechanical properties that can lead to an increase in fracture risk, as well as a delay in fracture repair (Blakytny et al., 2011; McCarthy et al., 2013). In addition, bone metabolism can also be secondarily affected by anti-diabetic pharmacological treatment causing detrimental or beneficial effects on this tissue (Molinuevo et al., 2010; Rzonca et al., 2004; Sedlinsky et al., 2011), thus contributing to further increases or decreases in fracture incidence.

In a recent meta-analysis, the gliptin family of DPP4 inhibitors as a whole has been suggested to reduce bone fractures (Monami et al., 2011). However, in their analysis these authors included not only results of patients in monotherapy with DPP4 inhibitors, but also of combined therapies with other anti-diabetic agents that have demonstrated effects on bone tissue. Additionally, although all DPP4 inhibitors share a common mechanism of action they also show structural heterogeneity (Gerich, 2010), and this might induce differences in certain side effects, for example on bone metabolism. Available information regarding the consequences of saxagliptin treatment on bone fracture incidence is sparse. On one hand, the prescription information for saxagliptin provided by the manufacturer informs that the incidence rate of bone fractures was 1.0 and 0.6 per 100 patient-years for saxagliptin and placebo respectively, in the pooled analysis of five 24-week trials with type 2 diabetic patients (Onglyza ${ }^{\circledR}, 2011$ ). This reported increase in fracture rate associated with saxagliptin treatment appears to be in agreement with the results of our present study. On the other hand, a recently published clinical trial was unable to find differences in bone fracture incidence between saxagliptin and placebo groups. However in that study, bone fracture was only evaluated as one of several safety endpoints, and patients included in the 2-year study were not stratified according to previous fractures, bone mineral density, use of antiosteoporotic medication or of add-on therapy with other antidiabetic agents that might secondarily affect bone metabolism and thus fracture incidence (such as thiazolidenediones or metformin) (Scirica et al., 2013).

There are multiple pathophysiological factors that could contribute to the Diabetes-associated impairment in bone quality, such as low levels of insulin or IGF1, or loss of sensitivity to their action (Blakytny et al., 2011; Merlotti et al., 2010). The roles of insulin and IGF1 in bone mass acquisition have been recognized for many years. Insulin and IGF1 can increase the proliferation of bone cells in culture as well as their expression of osteoblastic markers, and in vivo studies have demonstrated that deletion of the osteoblast insulin receptor reduces trabecular bone volume 
A

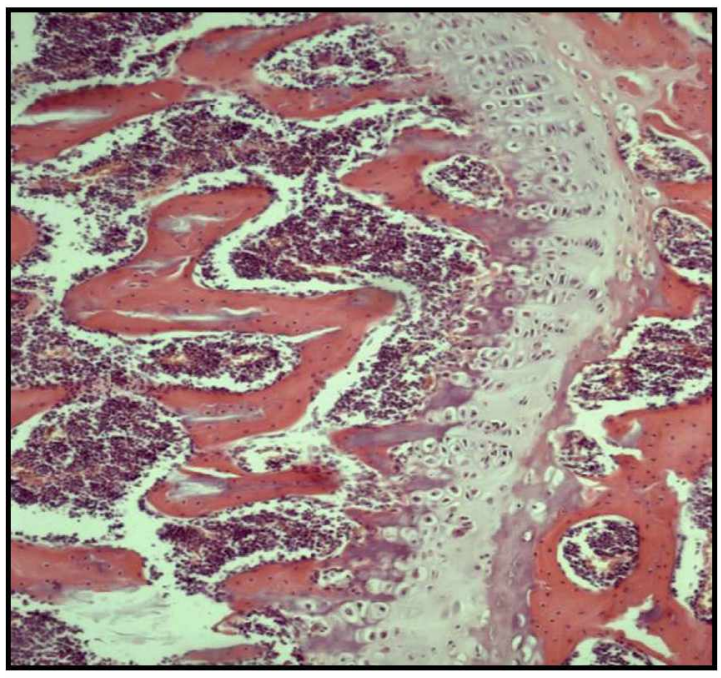

C

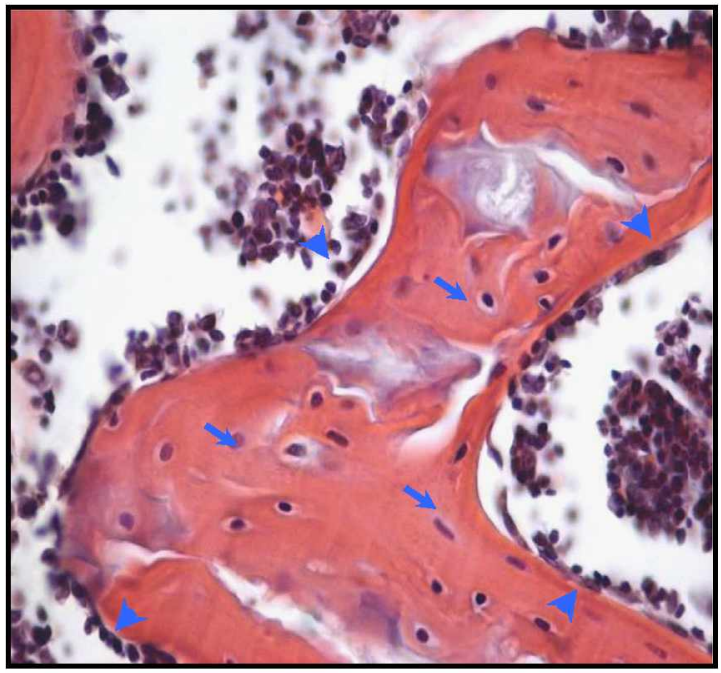

E

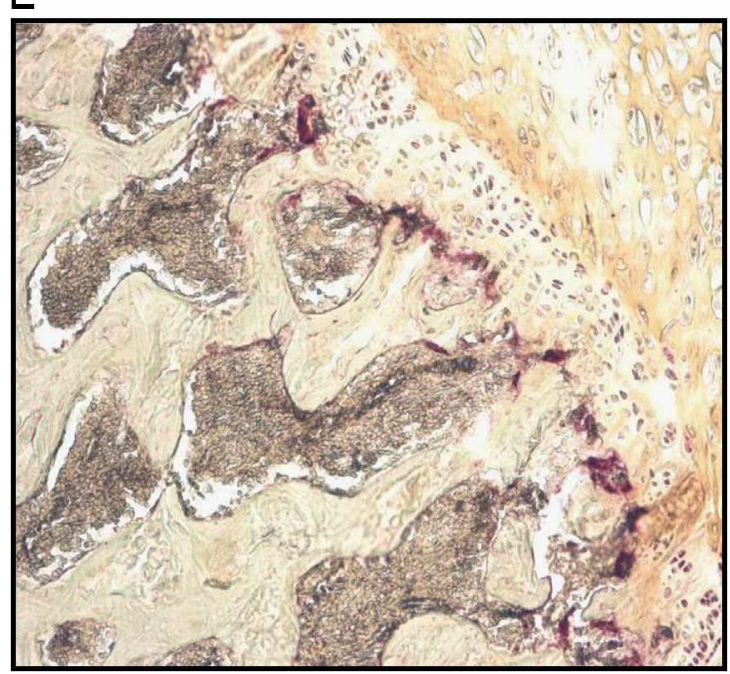

B

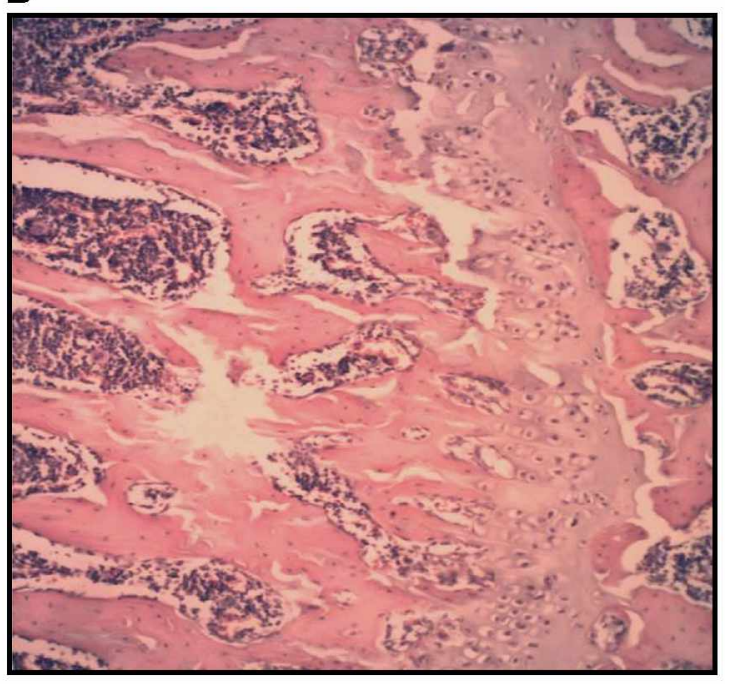

D

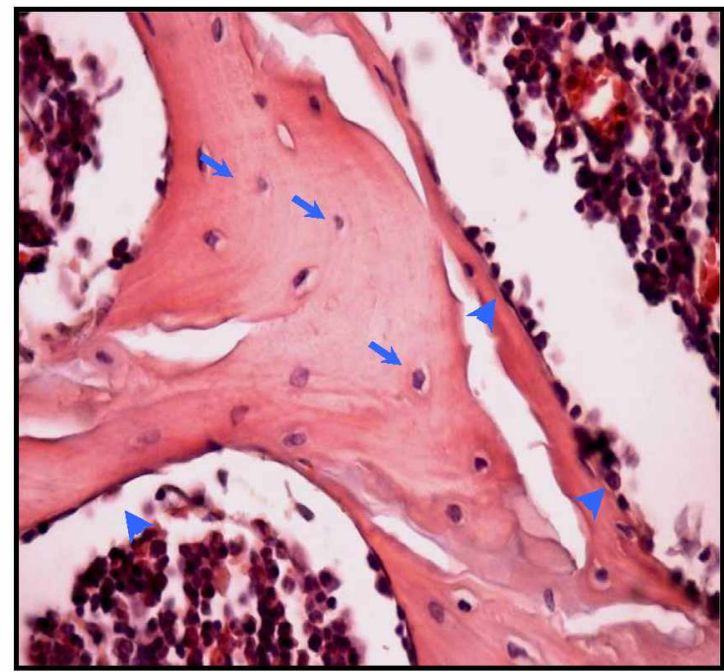

$F$

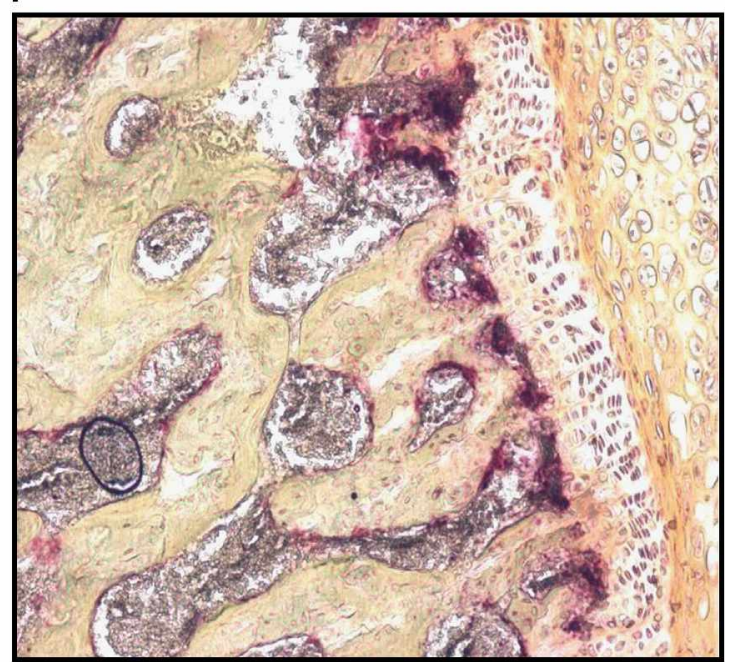

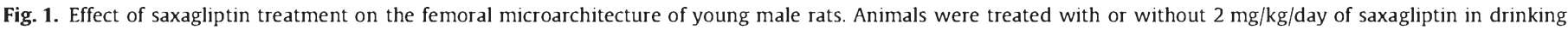

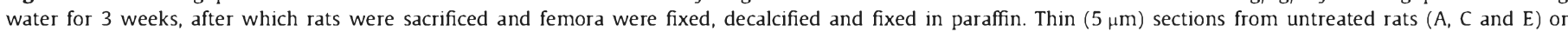

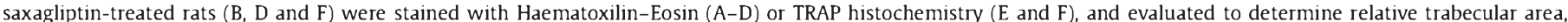

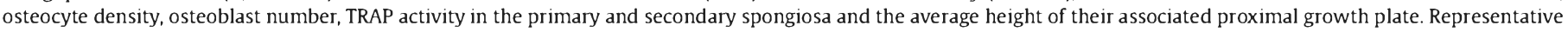
photographs are shown (A, B, E and F obj. $10 \times ; \mathrm{C}$ and D obj. $40 \times$ ). Arrows indicate osteocytes, arrowheads indicate bone surface osteoblastic cells 
Table 1

Effect of oral treatment with saxagliptin on femoral microarchitecture.

\begin{tabular}{lcc}
\hline & Control & Saxagliptin \\
\hline Relative trabecular bone [Tb.Ar/T.Ar] (\%) & $40 \pm 2$ & $43 \pm 2$ \\
Osteocyte density (N Ot/mm ${ }^{2}$ ) & $1787 \pm 163$ & $995 \pm 49$ \\
Osteoblast number (N Ob/mm) & $70 \pm 4$ & $47 \pm 3$ \\
Primary spongiosa relative TRAP( + ) area [TRAP.Ar] (\%) & $6.3 \pm 0.4$ & 0.01 \\
Proximal growth plate height $(\mu \mathrm{m})$ & $116 \pm 5$ & 0.01 \\
\hline
\end{tabular}

Data are expressed as the mean \pm S.E.M.
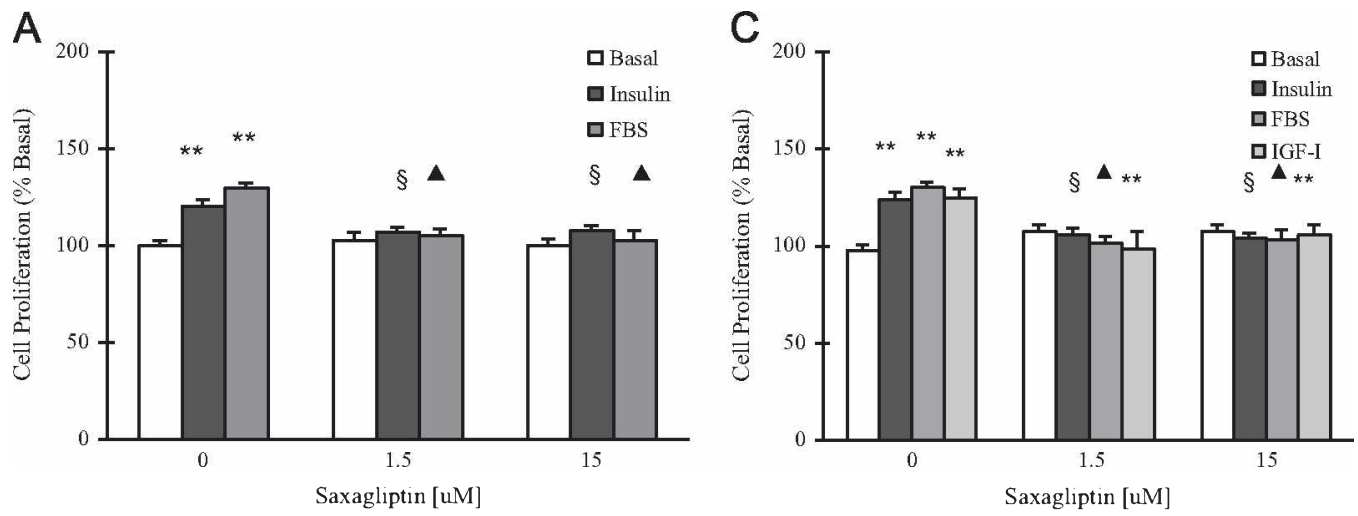

B

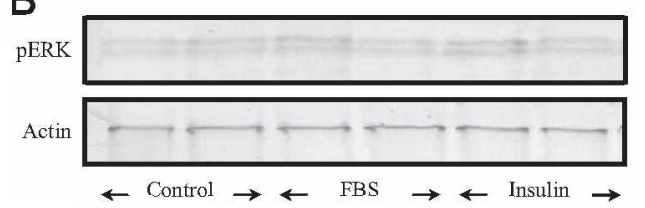

D
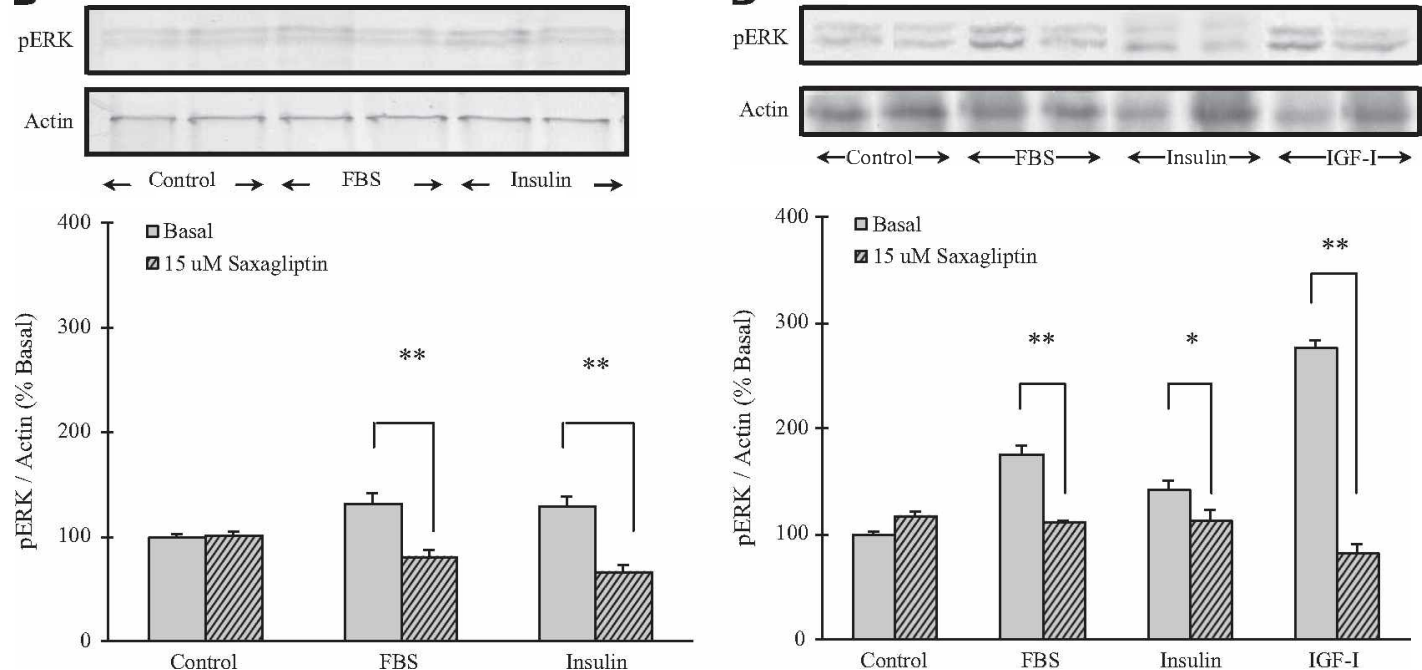

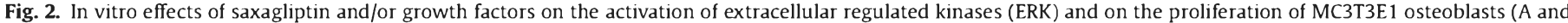

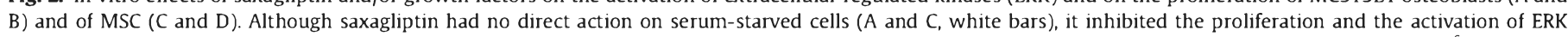

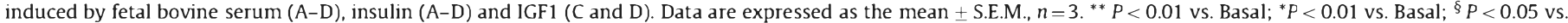
Insulin; $\triangle P<0.01$ vs. FBS; and $\bullet P<0.01$ vs. IGF.

(Fulzele and Clemens, 2012; Yang et al., 2010). After binding to their receptors, both insulin and IGF1 can recruit and activate common downstream signaling proteins such as phosphatidylinositol-3kinase and ERK in order to exert their anabolic effects on bone tissue (Taniguchi et al., 2006). In bone marrow progenitor cells (a pluripotent sub-class of MSC) ERK activation can induce the activation of Runx2, which in turn stimulates the expression of osteoblast-related genes such as type 1 collagen and osteocalcin (Franceschi and Xiao, 2003; Yang et al., 2010).

In the present study, in short-term $(1-24 \mathrm{~h})$ in vitro experiments we found that saxagliptin inhibited FBS-, insulin- and IGF1induced ERK phosphorylation and consequently MSC and osteoblastic proliferation. On the contrary, in the absence of serum or growth factors in the culture media saxagliptin had no effect either on ERK activation or on the proliferation of both cell types. Interestingly, different authors have found that for other cell types DPP4 activity is essential for growth factor-induced cell proliferation and extracellular matrix production (Kahne et al., 2000; Thielitz et al., 2008). In longer-term (2-3 weeks) in vitro studies with MSC and osteoblastic cells we demonstrated that, when added to osteogenic media that included FBS, saxagliptin dosedependently inhibited type 1 collagen secretion and mineralization, decreased the expression of osteocalcin and Runx2, and increased PPAR-gamma expression. This could be indicating a pro-adipogenic increase in the PPAR-gamma/Runx2 ratio induced by saxagliptin on MSC in vitro. However after 3 weeks of oral treatment with saxagliptin in vivo, we were unable to find a significant increase in bone marrow adiposity (data not shown). 
A

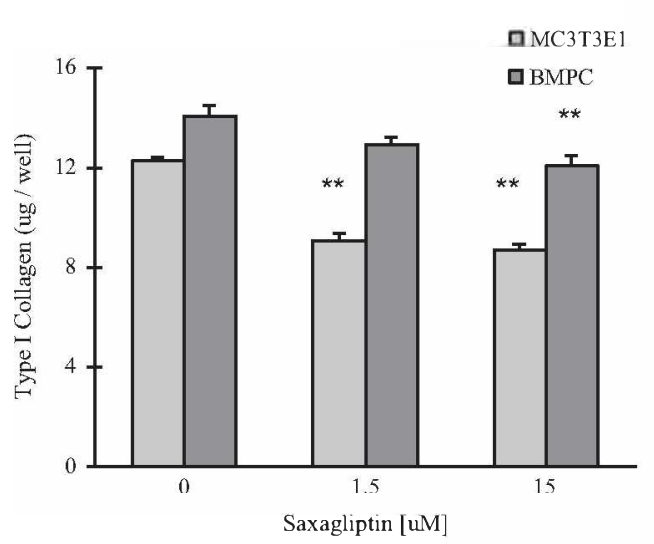

B

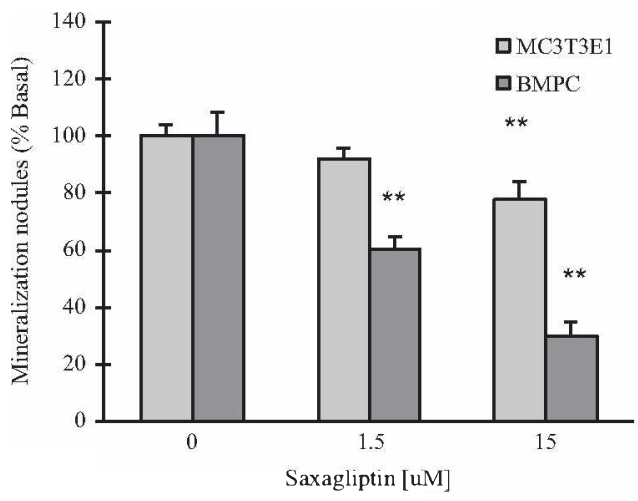

C

$\operatorname{Runx} 2$
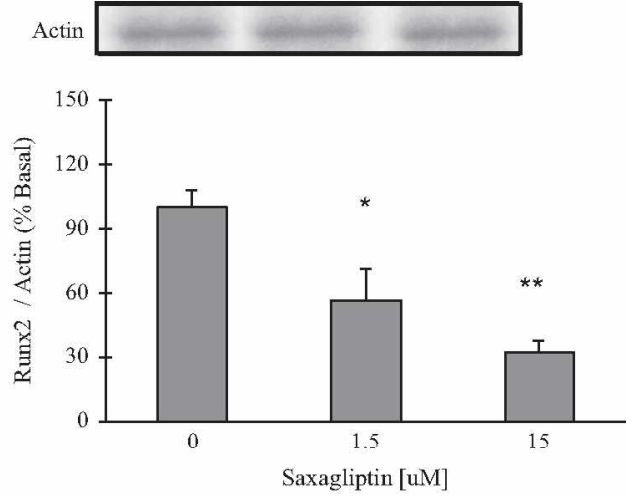

D

Osteocalcin

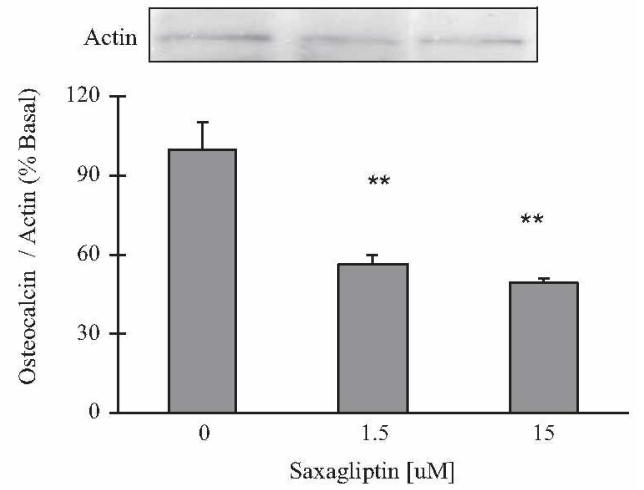

E PPAR gamma

Actin
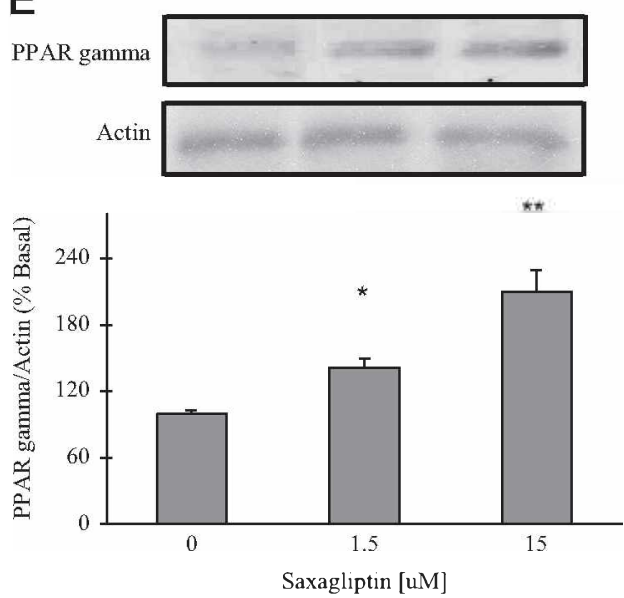

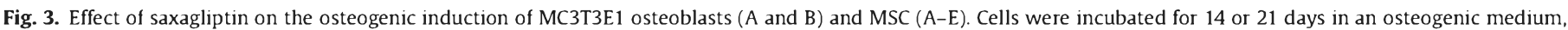

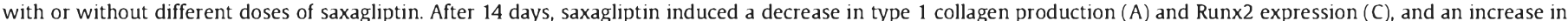

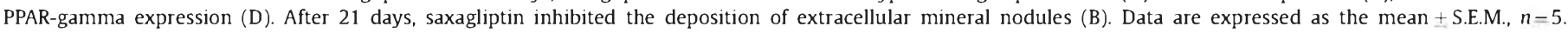
${ }^{* *} P<0.001$ vs. control; ${ }^{*} P<0.01$ vs. control.

In our study we chose to evaluate the action of saxagliptin on bone metabolism in young healthy insulin-sufficient male rats that are no longer in their rapid growth phase. We believe that this animal model has allowed us to adequately test our hypothesis, i.e. that this DPP4 inhibitor can simultaneously decrease the osteoblastic commitment and osteogenic potential of MSC, and induce deleterious changes in the microarchitecture of femoral trabecular bone. These results are in agreement with the reported clinical effects of saxagliptin on bone integrity (Onglyza ${ }^{\circledR}, 2011$ ), however our study presents limitations. Saxagliptin is usually prescribed as an adjunct therapy in adult patients with type 2 Diabetes whose pancreatic beta cells maintain a significant insulin-secretory response to increases in plasmatic incretin levels. In this context, our present results should provide the basis for future studies evaluating the action of saxagliptin (alone or in combination with other anti-diabetic drugs such as metformin) on bone metabolism in models of insulin-resistance and/or type 2 Diabetes. In addition, controlled clinical trials with specific bone alterations as pre-defined 
endpoints are necessary, in order to adequately verify the effects of saxagliptin on bone that have been informed by the manufacturer.

In conclusion, we have demonstrated deleterious effects of orally administered saxagliptin on rat femoral micro-architecture. These effects could be partially explained by the in vitro downregulation by saxagliptin of the ERK signaling pathway for growth factors such as insulin and IGF1 in MSC, which in turn could be mediating a decrease in the commitment of these cells to the osteoblastic phenotype.

\section{Author contributions}

Study design: ADM, MSM. Study conducted by: MLS, MSM, ADM. Data collection: MLS, MSM. Data analysis: MLS, MSM, ADM. Data interpretation: MLS, MSM, CS, LS, ADM. Drafting manuscript: MLS, MSM, ADM. Revising manuscript content: MLS, MSM, CS, LS, ADM. Approving final version of manuscript: MLS, MSM, CS, LS, ADM. ADM takes responsibility for the integrity of the data analysis.

\section{Acknowledgments}

This work was partially supported by Grants from Facultad de Ciencias Exactas, Universidad Nacional de La Plata (UNLP), Comisión de Investigaciones Científicas de la Provincia de Buenos Aires (CICPBA), Agencia Nacional de Promoción Científica y Tecnológica (PICT 1083 BID-1728/OC-AR) and Consejo Nacional de Investigación Científica y Tecnológica (CONICET) (PIP-0035). MLS is Doctoral Fellow of CICPBA. MSM is Investigador Adjunto of CONICET. ADM is a part-time Researcher and Professor of UNLP.

\section{References}

Ali, S., Fonseca, V., 2013. Saxagliptin overview: special focus on safety and adverse effects. Expert Opin. Drug Saf. 12, 103-109.

Blakytny, R., Spraul, M., Jude, E.B., 2011. The diabetic bone: a cellular and molecular perspective. Int. J. Low. Extrem. Wounds 10, 16-32.

Franceschi, R.T., Xiao, G., 2003. Regulation of the osteoblast-specific transcription factor, Runx2: responsiveness to multiple signal transduction pathways. J. Cell Biochem. 88, 446-454.

Fulzele, K., Clemens, T.L., 2012. Novel functions for insulin in bone. Bone 50, $452-456$.

Fura, A., Khanna, A., Vyas, V., Koplowitz, B., Chang, S.Y., Caporuscio, C., Boulton, D. W., Christopher, L.J., Chadwick, K.D., Hamann, L.G., Humphreys, W.G., Kirby, M., 2009. Pharmacokinetics of the dipeptidyl peptidase 4 inhibitor saxagliptin in rats, dogs, and monkeys and clinical projections. Drug Metab. Dispos. 37, 1164-1171.

Gagliardino, J.J., Santoro, S., Arellano, S., Di Girolamo, G., 2008. New treatments for type 2 diabetes mellitus: combined therapy with sitagliptin. Expert Opin. Pharmacother. 9, 1495-1507.

Gerich, J., 2010. DPP-4 inhibitors: what may be the clinical differentiators? Diabetes Res. Clin. Pract. 90, 131-140.
Guide, F.T.C.A.U.O.L.A., 2011. Committee for the Update of the Guide for the Care and Use of Laboratory Animals; Institute for Laboratory Animal Research Division on Earth and Life Studies, National Research Council of The National Academies, D.C, 2011 ed. The National Academic Press, Washington

Janghorbani, M., Van Dam, R.M., Willett, W.C., Hu, F.B., 2007. Systematic review of type 1 and type 2 diabetes mellitus and risk of fracture. Am. J. Epidemiol. 166, 495-505.

Kahne, T., Reinhold, D., Neubert, K., Born, I., Faust, J., Ansorge, S., 2000. Signal transduction events induced or affected by inhibition of the catalytic activity of dipeptidyl peptidase IV (DPP IV, CD26). Adv. Exp. Med. Biol. 477, 131-137.

Kanazawa, I., Yamaguchi, T., Yano, S., Yamauchi, M., Sugimoto, T., 2008. Metformin enhances the differentiation and mineralization of osteoblastic MC3T3-E1 cells via AMP kinase activation as well as eNOS and BMP-2 expression. Biochem. Biophys. Res. Commun. 375, 414-419.

Kyle, K.A., Willett, T.L., Baggio, L.L., Drucker, D.J. Grynpas, M.D., 2011. Differential effects of PPAR-\{gamma\} activation versus chemical or genetic reduction of DPP-4 activity on bone quality in mice. Endocrinology 152, 457-467.

Matteucci, E., Giampietro, O., 2009. Dipeptidyl peptidase-4 (CD26): knowing the function before inhibiting the enzyme. Curr. Med. Chem. 16, 2943-2951.

McCarthy, A.D., Molinuevo, M.S., Cortizo, A.M., 2013. AGEs and bone ageing in Diabetes mellitus. J. Diabetes Metab. 4, 6.

Merlotti, D., Gennari, L., Dotta, F., Lauro, D., Nuti, R., 2010. Mechanisms of impaired bone strength in type 1 and 2 diabetes. Nutr. Metab. Cardiovasc. Dis. 20, 683-690.

Molinuevo, M.S., Schurman, L., McCarthy, A.D., Cortizo, A.M., Tolosa, M.J., Gangoiti, M.V., Arnol, V., Sedlinsky, C., 2010. Effect of metformin on bone marrow progenitor cell differentiation: in vivo and in vitro studies. J. Bone Miner. Res. 25, 211-221.

Monami, M., Dicembrini, I., Antenore, A., Mannucci, E., 2011. Dipeptidyl peptidase-4 inhibitors and bone fractures: a meta-analysis of randomized clinical trials. Diabetes Care 34, 2474-2476.

Onglyza ${ }^{\circledR}$, s.p.i.D.B.-M.S., 2011. Onglyza ${ }^{\circledR}$ (saxagliptin) package insert, 2011 December. Bristol-Myers Squibb (packageinserts.bms.com/pi/pi_onglyza.pdf).

Rzonca, S.O., Suva, L.J., Gaddy, D., Montague, D.C., Lecka-Czernik, B., 2004. Bone is a target for the antidiabetic compound rosiglitazone. Endocrinology 145, 401-406.

Schon, E., Mansfeld, H.W., Demuth, H.U., Barth, A., Ansorge, S., 1985. The dipeptidy peptidase IV, a membrane enzyme involved in the proliferation of $\mathrm{T}$ lymphocytes. Biomed. Biochim. Acta 44(K9-15).

Scirica, B.M., Bhatt, D.L., Braunwald, E., Steg, P.G., Davidson, J., Hirshberg, B., Ohman, P., Frederich, R., Wiviott, S.D., Hoffman, E.B., Cavender, M.A., Udell, J.A., Desai, N. R., Mosenzon, O., McGuire, D.K., Ray, K.K., Leiter, L.A., Raz, I., 2013. Saxagliptin and cardiovascular outcomes in patients with Type 2 Diabetes Mellitus. N. Engl. J. Med. 369, 1317-1326.

Sedlinsky, C., Molinuevo, M.S., Cortizo, A.M., Tolosa, M.J., Felice, J.I., Sbaraglini, M.L., Schurman, L., McCarthy, A.D., 2011. Metformin prevents anti-osteogenic in vivo and ex vivo effects of rosiglitazone in rats. Eur. J. Pharmacol. 668, 477-485.

Stanley, K.T., VanDort, C., Motyl, C., Endres, J., Fox, D.A., 2006. Immunocompetent properties of human osteoblasts: interactions with $\mathrm{T}$ lymphocytes. J. Bone Miner. Res. 21, 29-36.

Tahara, A., Matsuyama-Yokono, A., Nakano, R., Someya, Y., Hayakawa, M., Shibasaki, M., 2009. Antihyperglycemic effects of ASP8497 in streptozotocin-nicotinamide induced diabetic rats: comparison with other dipeptidyl peptidase-IV inhibitors. Pharmacol. Rep. 61, 899-908.

Taniguchi, C.M., Emanuelli, B., Kahn, C.R., 2006. Critical nodes in signalling pathways: insights into insulin action. Nat. Rev. Mol. Cell Biol. 7, 85-96.

Thielitz, A., Vetter, R.W., Schultze, B., Wrenger, S., Simeoni, L., Ansorge, S., Neubert K., Faust, J., Lindenlaub, P., Gollnick, H.P., Reinhold, D., 2008. Inhibitors of dipeptidyl peptidase IV-like activity mediate antifibrotic effects in normal and keloid-derived skin fibroblasts. J. Invest. Dermatol. 128, 855-866.

Yang, J., Zhang, X., Wang, W., Liu, J., 2010. Insulin stimulates osteoblast proliferation and differentiation through ERK and PI3K in MG-63 cells. Cell Biochem. Funct. $28,334-341$. 